\title{
Linearization strategies during language production
}

\author{
FERNANDA FERREIRA and JOHN M. HENDERSON \\ Michigan State University, East Lansing, Michigan
}

\begin{abstract}
The two experiments reported here were designed to examine discourse planning during language production. Following the work of Levelt (1981, 1982), participants were shown simple networks consisting of two branches, and their task was to describe the networks. The two branches of the networks differed in either length or complexity; the dependent measure was participants' decisions to describe the left or right branch first. The experiments showed that speakers preferred to describe a less complex branch before a more complex branch and preferred to describe a shorter branch before a longer branch. Levelt's minimal-load principle is invoked to explain these results, along with the principle of incrementalism (Levelt, 1989). Discussion focuses on the possibility that incrementalism in production is useful to both the speaker and the listener.
\end{abstract}

To produce any multiword utterance, the speaker must decide in what order words, phrases, and clauses are to be produced. Recent work in language production has uncovered some of the processes underlying decisions about word order in sentences. For example, Bock and colleagues (Bock, 1986, 1987a, 1987b; Bock \& Warren, 1985; Kelly, Bock, \& Keil, 1986) have shown that words that are frequent, prototypical, concrete, or semantically primed tend to take earlier positions in a sentence. Ferreira (1994) has demonstrated that thematic roles such as agent and experiencer tend to be subjects and that roles such as theme and patient tend to be objects. Other work has shown that the subject of a sentence is usually the topic under discussion (e.g., Tomlin, 1983). The general principle seems to be that the more available or salient a concept is, the earlier in a sentence the corresponding linguistic constituent tends to be.

Although researchers are beginning to understand how word order within clauses is decided, little is known about how speakers order clauses within larger discourses - that is, about how speakers adopt a linearization strategy (Levelt, 1981, 1982, 1989). For some domains of discourse,

The research reported here was supported by a grant to the first author from the Natural Sciences and Engineering Research Council of Canada and by the Department of Psychology at the University of Alberta. Manuscript preparation was supported by National Science Foundation Grant SBR-9319272 to the first author. The authors thank Lucille Lavender for assistance with preparation of stimuli and testing of participants for the experiments, Amy Adkins and Melissa Johnson for conducting some of the data analyses, and Kay Bock and two anonymous reviewers for their comments on a previous version of this article. The authors also thank the Laboratory of Experimental Psychology, University of Leuven, for providing a warm and collegial environment during the writing phase of this research. Correspondence should be addressed to either author, Department of Psychology, Michigan State University, East Lansing, MI 48824-1117 (e-mail: fernanda@pilot.msu.edu, orjohn@eyelab.msu.edu).

-Accepted by previous editor, Geoffrey R. Loftus the appropriate linearization strategy is obvious. For example, a speaker wishing to describe a trip that he or she had recently taken would most likely order the clauses temporally, so that events that occurred earlier would be described before those that occurred later. In such domains, the linearization strategy is a direct reflection of the temporal structure of the underlying events. As Levelt (1989) notes, the assumption that clauses should be ordered temporally is strong enough that most comprehenders will draw very different inferences about the quality of the hospital in question from the two sentences Bill was shot and went to the local hospital and Bill went to the local hospital and was shot.

For other types of discourse, such as descriptions of spatially organized structures, the proper ordering scheme is less obvious. But as Levelt observed, when speakers describe structures such as their offices or homes-structures lacking temporal constraints-they do not order clauses randomly. Instead, they impose an ordering strategy onto the domain. Most speakers will describe their home by taking the listener on a mental "tour," beginning at the entrance, and then proceeding through each room as if they were actually walking through it. Such a strategy is of benefit to both the speaker and the listener: It allows the speaker to keep track of what has been said and what remains to be described, and a clear linearization scheme allows the listener to accurately reconstruct the domain of the discourse. Clearly, it is easier to form a representation of someone's house if the description takes the form of an abstract tour rather than a series of random jumps (see Lloyd, 1991, for a demonstration of this point with children and adults).

However, because of the presence of choice points, it is not always possible to take the listener on a simple "linear" walk through a house. For example, one may begin by describing the front door of one's home and then have to decide whether to describe the living room on the left, the den on the right, or the staircase directly in front. The 
issue, then, is what are the decision principles that the language production system obeys when it is faced with these choice points?

Levelt $(1981,1982)$ examined this issue using networks of colored circles connected by lines, illustrated in Figure 1. The speaker begins his or her description at the circle above the arrow. At the circle immediately above this starting circle, the speaker must decide whether to describe the left or the right branch first. The variable of interest is the participant's left/right decision. Levelt varied a number of characteristics of the two branches. We will focus on two in this paper. First, the branches could differ in length, as shown in Figure 1A. Second, the branches could differ in complexity, as shown in Figure 1B. We will call the type of branch on the left of Figure $1 \mathrm{~B}$ a linear branch, because the speaker can describe the branch by mentioning each circle sequentially (thus, both branches of Figure 1A are also linear). We will call the branch on the right side of Figure $1 \mathrm{~B}$ a choice branch, because the speaker must make a decision at Circle 8 about whether to describe Circle 9 or Circle 10 first.

Of relevance to this study are two findings obtained by Levelt: First, participants preferred to describe shorter branches before describing longer ones. Thus, for Figure 1A, speakers tended to describe the left branch first. Second, when branch length was equated, linear branches were described before choice branches. Thus, for Figure $1 \mathrm{~B}$, speakers tended to describe the left side before the right. Levelt (1989) proposed a single principle to explain both of these findings, which he termed the minimalload principle: Speakers attempt to minimize the memory load from "return addresses." A return address is any circle that defines a choice point. Thus, Circle 2 in Fig-

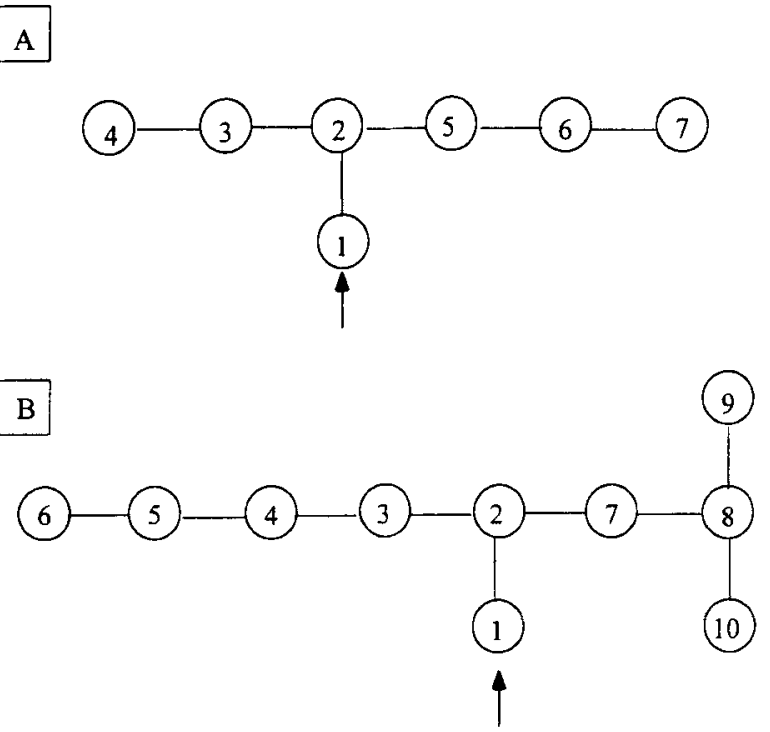

Figure 1. Examples of two figures used by Levelt (1981). Panel A consists of a short branch on the left and a longer branch on the right; panel $B$ consists of a left linear and a right choice branch, both of the same length. ure $1 \mathrm{~A}$ is a return address, as are Circles 2 and 8 in Figure $1 \mathrm{~B}$.

The minimal-load principle explains the preference for short branches as follows: If the speaker begins with the shorter branch, the return address (Circle 2) need only be maintained during the description of two circles. If the speaker begins with the longer branch, the return address must be maintained during the description of three circles. The minimal-load principle predicts that speakers will prefer to describe the shorter branch first. The minimal-load principle can also explain the preference to describe linear branches before choice branches. Consider Figure 1B once again. If the speaker describes the left branch first, the return address represented by Circle 2 must be maintained in memory, but then it can be released before the return address represented by $\mathrm{Cir}$ cle 8 must be stored. If the speaker were to describe the right branch first, Circle 2 would have to be stored, and then Circle 8 would have to be stored before Circle 2 had been released. Describing the choice branch before the linear branch requires that two return addresses be maintained in memory during the description of the network; however, if the linear branch is described first, only one return address need be maintained at any point. Levelt's $(1981,1982)$ work shows that the language production system prefers to keep as few return addresses in mind as possible and for as brief a time as possible.

Even though the studies conducted by Levelt (1981, 1982) have uncovered important principles underlying discourse production (one of which, the minimal-load principle, is to be investigated in this study), numerous questions remain to be addressed. First, although we know from Levelt's work that linear branches are preferred over choice branches and short branches over long branches, we do not know how the language production system balances these two preferences. In Experiment 1, we found that the preference for linear branches is stronger than the preference for short branches, and we suggest that this effect can be explained if we assume that speakers do not base their decision concerning which branch to describe first on plans spanning the domain of an entire network, but instead plan only over the domain of a single branch. This suggestion was tested directly in Experiment 2: By introducing a variation to Levelt's original procedure, we show that memory load is computed only over single branches, not entire networks. Together, the two experiments indicate that discourse planning conforms to Levelt's (1989) principle of incrementalism in language production.

\section{EXPERIMENT 1}

In Experiment 1, we investigated the minimal-load principle by examining how participants' decisions to begin their network descriptions with the left-hand or right-hand branch of the networks would be affected by both the type and length of branch. First, we varied whether the left side of the initial choice circle was lin- 
ear or choice. In the linear condition, the circles were arranged so that one circle was connected to just one other circle. In the choice condition, one of the circles was connected to two other circles, requiring the speaker to decide which one to describe first. The right side of the initial choice circle could also be either linear or choice. Finally, all networks consisted of three circles on one side and five on the other, or vice versa.

\section{Method}

Participants. Twenty-four University of Alberta undergraduates participated in the experiment in exchange for partial credit in their introductory psychology courses. All participants were unaware of the issues under consideration in the experiment, had normal color vision, and considered English their dominant language.

Materials. Twenty-four networks, such as the ones shown in Figure 2 , were created. The networks were drawn on eight $81 / 2 \times 11$ in. cards. The circles were $1.4 \mathrm{~cm}$ in diameter and were connected by lines $3 \mathrm{~cm}$ in length. On any one network, each circle was of a unique color. The networks appeared in one of eight conditions: (1) linear3-linear5, left branch linear, three circles-right branch linear, five circles; (2) linear5-linear 3, left branch linear, five circlesright branch linear, three circles; (3) linear3-choice5, left branch linear, three circles-right branch choice, five circles; (4) linear 5 choice 3 , left branch linear, five circles-right branch choice, three circles; (5) choice3-linear5, left branch choice, three circles-right branch linear, five circles; (6) choice5-linear 3 , left branch choice, five circles-right branch linear, three circles; (7) choice 3-choices, left branch choice, three circles-right branch choice, five circles; (8) choice5-choice 3, left branch choice, five circles-right branch choice, three circles.

For each of the eight conditions, three different networks were drawn (differing in the colors of the circles). The networks were created so that linear branches contained a bend or two (depending on the length of the figure) in order to make them visually similar to the choice branches.

Eighteen filler networks were also created. The fillers were similar to the experimental networks except that the two branches contained an equal number of circles, and all branches were linear. These features of the fillers were intended to prevent the participants from expecting the same type of network on each trial of the experiment. The 42 ( 24 experimental plus 18 filler) networks were<smiles>OOOOOOC(O)OOO</smiles>

Linear-Linear<smiles>COC(OOOO)OOOO</smiles>

Choice-Linear

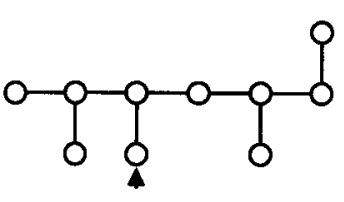

Choice-Choice

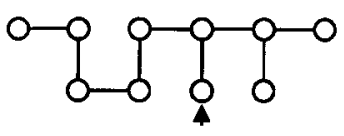

Linear-Choice
Figure 2. Examples of the networks used in the experiments. Each network also occurred in its mirror-reversed version, for a total of eight conditions. arranged in three random orders. (The appropriate order for a given participant was generated before the experimental session.)

Procedure. The participants were run individually in a small quiet room. They were shown a sample network similar to the filler networks and were told that their task was to describe a number of such networks. They were asked to describe the networks so that someone else could later draw them from their descriptions alone. The participants were asked to refer to the circles by color and to call the circles "dots" (e.g., "the yellow dot"). The color was requested from the participant because color uniquely identified each circle, and the word dot was requested because it begins and ends with a stop consonant, a feature that could be useful if we at any point chose to perform waveform analyses on the participants' productions (we have not done so up to this point). No other constraints were placed on the participants' productions.

The procedure was as follows. First, the experimenter placed in front of the participant a card with all the colors used for the circles and their corresponding labels. He or she was asked to examine the card and to become familiar with the terms. The purpose of this step in the procedure was to ensure that the participants would have a readily available term for each color used in the networks. For example, the color for which we chose the label mauve can be referred to by a number of other terms.

At this point, the tape recorder was turned on, and the participant was given three practice trials. A trial consisted of the following events. First, the experimenter placed a single card face down in front of the participant. Next, the experimenter turned over the card and said "begin." The participant was told to begin with the circle immediately above the arrow (see Figure 2). The participants were also told to proceed at their own pace and to speak as if they were in a normal conversation. They were free to take a break at any point between trials. During the practice trials, the experimenter monitored the participant's performance and corrected any deviations from the instructions (e.g., use of an inappropriate color term). Any questions from the participant were answered, and then he or she was told that the "real" experiment would begin. The participant then proceeded through the 42 trials.

Data analysis. The design of the experiment was 2 (left side linear vs. left side choice) $\times 2$ (right side linear vs. choice) $\times 2$ (3 vs. 5 circles), with all variables within participants. The dependent measure was the decision to go left or right immediately after the circle directly above the starting circle. A left decision was scored as a 0 , and a right decision as a 1 . Thus, mean values below .5 are leftward biases, and those above .5 are rightward biases.

\section{Results}

Six of the 24 participants consistently chose the same side of every network first ( 2 participants always went left first, and 4 always went right first). An analysis of variance was conducted both on the data from all 24 participants and on the data excluding those 6 participants. The results were virtually identical, so reported results are based on only the 18 participants who varied in their initial choice. There was no effect of which of the three random orders was used, and so this variable will not be discussed further. All results were significant at .05 , unless otherwise indicated.

The results are shown in Table 1. When the two branches were of the same type (i.e., linear-linear or choicechoice), then the participants' decisions were based on number of circles (shorter side described first). When the branches were of different types (linear-choice or choice-linear), then number of circles ceased to matter, and the decision was to describe the linear branch first. 
Table 1

The Participants' Decisions to

Describe the Left Branch (Values < .50) or the

Right Branch (Values $>$.50) First in Experiment 1

\begin{tabular}{lcc} 
& \multicolumn{2}{c}{ Number of Circles } \\
\cline { 2 - 3 } Branch Type & 3 left, 5 right & 5 left, 3 right \\
\hline Linear-Linear & .26 & .72 \\
Choice-Choice & .28 & .80 \\
Linear-Choice & .35 & .30 \\
Choice-Linear & .83 & .80 \\
\hline
\end{tabular}

Note-SEM $=0.077$.

Thus, the preference for a linear over a choice branch was stronger than the preference for a shorter over a longer branch.

These conclusions were supported by the following statistical results: First, there was a main effect of whether the left side was linear or choice $[F(1,17)=20.48]$ : The participants tended to go left when the left side was linear (.41) and right when it was choice (.68). The main effect of whether the right side was linear or choice was also reliable $[F(1,17)=11.94]$. The participants preferred to go right when the right side was linear (.65) and left when it was choice (.43). There was also a main effect of number of circles $[F(1,17)=10.17]$. The participants preferred to go left when the number of circles was three on the left and five on the right (.43), and they preferred to go right in the reverse situation (.65).

More importantly, the three-way interaction among these three factors was reliable $[F(1,17)=24.23]$. The pattern, which is shown in Table 1, demonstrates that the participants preferred to describe the shorter side first only when the type of branch on each side was the same; otherwise, they preferred to describe the linear before the choice branch. No other effects were significant (all $p \mathrm{~s}>.10$ ).

To explore the strategies for describing these networks further, we examined how the participants made their way from the end of the first branch, regardless of what form it took, to the start of the second branch. One strategy that the participants could adopt is what we will call a stepping strategy (and the participants adopting this strategy will be termed steppers): They could move sequentially, circle by circle, from the last circle of the first described branch to the return address, mentioning each intervening circle on the way. We will refer to the other strategy as jumping (and the participants adopting this strategy will be termed jumpers): The participants could jump from the last circle of the first described branch to the return address, ignoring all intervening circles. In Experiment 1,22 of the 24 participants jumped on every single trial. One of the remaining 2 participants used the stepping strategy on about half the practice trials and on the first experimental network, but then abandoned that strategy in favor of jumping for the remainder of the networks. The other participant stepped on every trial of the experiment. Interestingly, his description style was noticeably different from the others'. Most participants spoke in sentence-like utterances, such as the following: "We begin with a dark blue dot. Above the dark blue dot is a yellow dot. To the left of the yellow dot is a light blue dot." In contrast, the uniform stepper spoke rapidly, and his utterances took this form: "Dark blue dot up to yellow dot left to light blue dot ..." and so on. Apparently, the stepper took a rather mechanical approach to his task and treated the circles almost like parts of a list. Of course, it is difficult to draw any conclusions on the basis of this 1 participant; we will discuss stepping in more detail later, since the stepping strategy was more common in Experiment 2.

\section{Discussion}

Experiment 1 made two clear points: First, speakers prefer to begin their network descriptions with the linear branch rather than the choice branch. Second, if the branches are of the same type, then speakers prefer to begin their descriptions with the shorter branch. The question we will now address is, are these findings consistent with the minimal-load principle?

To address this question, we must make the predictions of the minimal-load principle more explicit. To do so, we constructed charts like the ones used by Levelt (1982), and these are shown in Figure 3. These charts were constructed on the basis of the assumption that the hypothetical speaker is a jumper. This assumption does not affect the predicted pattern of results but only subtracts a constant from the memory load assumed for each network (i.e., steppers have a slightly higher memory load for any network because they must keep track of any return addresses as they proceed circle by circle to those addresses).

First, let us consider the networks that differed only in number of circles (the networks for which both branches were either linear or choice). Focus first on the top half of Figure 3, which shows a linear-linear network (the same logic applies for the choice-choice networks, and so these will not be discussed). The only return address for this network is Circle 2. If speakers begin with the shorter branch, they must keep Circle 2 in memory during the description of three circles, for a total load of three. If speakers begin with the longer branch, they must keep Circle 2 in memory during the description of five circles, for a total load of five. Thus, the memory load is three for the network description in which the short branch is described first, but it is five if the long branch is described first (see lines labeled "Network ML"). The minimal-load principle makes the correct prediction that the short branch will be described before the long one.

Now let us turn to the networks that show the dominance of the decision based on type of branch over the decision based on length - the networks with a short choice branch and a longer linear branch (lower half of Figure 3). As can be seen under the heading, "Experiment 1: Do not return to initial entry point" (the lines labeled "Network ML"), the minimal-load principle incorrectly predicts that there will be no preference to go left 


\section{Linear-Linear}

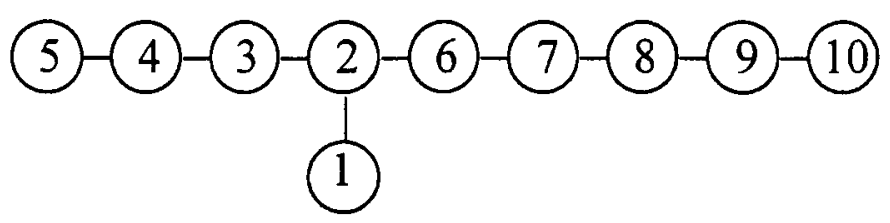

Experiment 1: Do not return to initial entry point

\begin{tabular}{|c|c|c|c|c|c|c|c|c|c|c|c|c|c|c|c|}
\hline \multirow[t]{3}{*}{ Short side 1st } & Circles described & 1 & 2 & 3 & 4 & 5 & 2 & 6 & 7 & 8 & 9 & 10 & & & \\
\hline & Network ML & 0 & 0 & 1 & 1 & 1 & 0 & 0 & 0 & 0 & 0 & 0 & $=3$ & & \\
\hline & Branch ML & & 0 & 1 & 1 & 1 & 0 & & & & & & $=3$ & & \\
\hline \multirow{4}{*}{ Long side 1st } & Circles described & 1 & 2 & 6 & 7 & 8 & 9 & 10 & 2 & 3 & 4 & 5 & & & \\
\hline & Network ML & 0 & 0 & 1 & 1 & 1 & 1 & 1 & 0 & 0 & 0 & 0 & $=5$ & & \\
\hline & Branch ML & & 0 & 1 & 1 & 1 & 1 & 1 & 0 & & & & $=5$ & & \\
\hline & Experi & it 2 & $\operatorname{Re}$ & turn & to in & nitial & I ent & ry pc & & & & & & & \\
\hline \multirow[t]{3}{*}{ Short side 1st } & Circles described & 1 & 2 & 3 & 4 & 5 & 2 & 6 & 7 & 8 & 9 & 10 & 2 & 1 & \\
\hline & Network ML & 0 & 0 & 1 & 1 & 1 & 0 & 1 & 1 & 1 & 1 & 1 & 0 & 0 & $=8$ \\
\hline & Branch ML & & 0 & 1 & 1 & 1 & 0 & & & & & & & & $=3$ \\
\hline \multirow[t]{3}{*}{ Long side 1st } & described & 1 & 2 & 6 & 7 & 8 & 9 & 10 & 2 & 3 & 4 & 5 & 2 & 1 & \\
\hline & Network ML & 0 & 0 & 1 & 1 & 1 & 1 & 1 & 0 & 1 & 1 & 1 & 0 & 0 & $=8$ \\
\hline & Bran & & 0 & 1 & 1 & 1 & 1 & 1 & 0 & & & & & & $=5$ \\
\hline
\end{tabular}

\section{Linear-Choice}

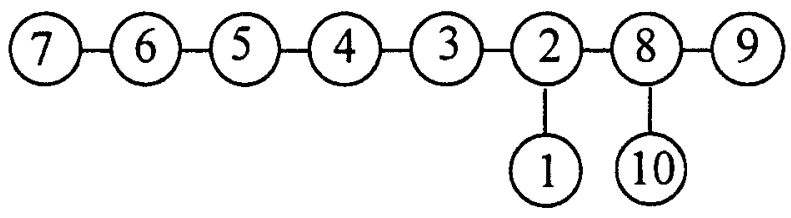

Experiment 1: Do not return to initial entry point

\begin{tabular}{|c|c|c|c|c|c|c|c|c|c|c|c|c|c|c|c|c|}
\hline Linear side 1st & Circles described & 1 & 2 & 3 & 4 & 5 & 6 & 7 & 2 & 8 & 9 & 8 & 10 & & & \\
\hline & Network ML & 0 & 0 & 1 & 1 & 1 & 1 & 1 & 0 & 0 & 1 & 0 & 0 & $=6$ & & \\
\hline & Branch ML & & 0 & 1 & 1 & 1 & 1 & 1 & 0 & & & & & $=5$ & & \\
\hline Choice side 1st & Circles described & 1 & 2 & 8 & 9 & 8 & 10 & 2 & 3 & 4 & 5 & 6 & 7 & & & \\
\hline & Network ML & 0 & 0 & 1 & 2 & 1 & 2 & 0 & 0 & 0 & 0 & 0 & 0 & $=6$ & & \\
\hline & Branch ML & & 0 & 1 & 2 & 1 & 2 & 0 & & & & & & $=6$ & & \\
\hline & Experi & te $2:$ & $\operatorname{Re}$ & urn & to in & itial & entr & $\mathrm{pc}$ & & & & & & & & \\
\hline Linear side 1st & Circles described & 1 & 2 & 3 & 4 & 5 & 6 & 7 & 2 & 8 & 9 & 8 & 10 & 2 & 1 & \\
\hline & Network ML & 0 & 0 & 1 & 1 & 1 & 1 & 1 & 0 & 1 & 2 & 1 & 2 & 0 & 0 & $=11$ \\
\hline & Branch ML & & 0 & 1 & 1 & 1 & 1 & 1 & 0 & & & & & & & $=5$ \\
\hline Choice side 1 st & Circles described & 1 & 2 & 8 & 9 & 8 & 10 & 2 & 3 & 4 & 5 & 6 & 7 & 2 & 1 & \\
\hline & Network ML & 0 & 0 & 1 & 2 & 1 & 2 & 0 & 1 & 1 & 1 & 1 & 1 & 0 & 0 & $=11$ \\
\hline & Branch ML & & 0 & 1 & 2 & 1 & 2 & 0 & & & & & & & & $=6$ \\
\hline
\end{tabular}

Figure 3. Illustration of the predictions for Experiment 2. ML = memory load; bold numbers stand for a return address circle.

or right for these networks. Whether the choice side or the linear side is described first, the total memory load for this network is six. Thus, some revision to the minimalload principle appears to be necessary to account for the finding from Experiment 1 that embedness carries more weight than greater length.

One possibility is to consider whether the memory load computed by speakers prior to beginning their descrip- tions is based on the entire network, as has been assumed up to this point, or rather is based on just part of the network. The assumption that memory load is computed for the entire network underlies the charts we have discussed up to this point. On this assumption, speakers plan one entire network description and compute the corresponding memory load, they then plan the other network description and compute its corresponding memory load, 
and they then choose the less memory-demanding description. Note that, according to this view, the speaker does the work of planning two entire descriptions before beginning to speak. The question arises whether the speaker really saves any effort by engaging in this strategy, given that any savings gained by articulating the simpler network description might be offset by the work required to plan two entire network descriptions.

An alternative conception of discourse planning for these networks is that speakers compute memory load for each branch of the network, compare the difficulty of the two, and then begin their descriptions with the less memory-demanding branch. This possibility is shown in Figure 3, on the lines labeled "Branch ML." Consider the linear-linear network first and only the top half of the figure (labeled "Experiment 1: Do not return to initial choice point"). The description of the short branch includes the descriptions of Circles 2,3 , and 4 ; the description of the long branch includes the description of Circles $2,6,7,8,9$, and 10 . The memory load for the short branch is three, whereas that for the long branch is five. Thus, the view that speakers compute memory load only over the domain of a branch correctly predicts the results obtained in Experiment 1: Speakers should prefer to begin their descriptions with the short branch. (The same logic applies for the choice-choice networks.)

Now let us turn to the linear-choice figure. The description of the linear branch includes the description of Circles 2, 3, 4, 5, 6, and 7; the description of the choice branch includes the description of Circles 2, 8,9,8, and 10. As can be seen in the first half of the figure on the lines labeled "Branch ML," the memory load for the linear branch alone is five, and that for the choice branch alone is six. Therefore, if we assume that speakers compute only the complexity of each branch and then begin their description with the simpler one, we correctly predict the pattern of results obtained in Experiment 1: Speakers should prefer to begin their descriptions with the less complex branch, even though it is longer than the choice branch. Embedness is weighted more heavily than greater length because the memory load associated with beginning with the choice, shorter branch is higher than the memory load associated with beginning with the linear, longer branch.

It is important to note that the values for memory load should only be interpreted as ordinal values; if the value for beginning with a particular branch is higher than the value for beginning with the other branch, then we assume that the strategy with the higher value carries a greater memory load, and so the branch associated with the greater load will be avoided. Therefore, at this point in our work on linearization strategies, it does not matter whether the load for one strategy is five and the other six or whether the load for one strategy is five and the other seven - no inferences should be made from the size of the load differences. We wish to avoid such inferences because it not clear what effect additional memory load causes (i.e., whether effects are additive or multiplicative).

To provide a direct test of the hypothesis that speakers compute memory load only over a single branch, we con- ducted a second experiment with a minor modification to the task the speakers were asked to perform. Rather than having speakers start at Circle 1 and then Circle 2, describe one branch, describe the other branch, and then stop, we required them to start at Circle 1 and then at Circle 2, describe one branch, describe the other branch, return to Circle 2, and then exit through Circle 1. One consequence of this change is that it forces the speaker to keep Circle 2 as a return address while both the first and second branches are being described.

This change in procedure allows us to contrast the predictions of the two views of planning we described above: the view that planning occurs over the domain of the entire networks, and the view that planning occurs only over the domain of single branches. Consider first the linear-linear network in the top half of Figure 3. If the speaker must return to the return address represented by Circle 2 (see the lower half of the figure labeled "Experiment 2: Return to initial entry point"), and if speakers base their decisions on the complexity of entire network descriptions, then the memory load is the same regardless of which branch is described first (see the line labeled "Network ML"). As a result, this view predicts that speakers will have no preference to begin with one branch or the other. The same prediction holds for the linear-choice networks, as shown in the bottom half of Figure 3 (again, the line labeled "Network ML"): Speakers should show no preference to describe one branch before the other.

In contrast, if speakers base their decisions on the complexity of just the individual branches, then they are predicted to show the same preferences in Experiment 2 that they showed in Experiment 1. Consider Figure 3 again, focusing particularly on the section labeled "Experiment 2: Return to initial entry point." For the linearlinear network, the memory load for the shorter branch is less than the memory load for the longer branch (see "Branch ML"). Similarly, for the linear-choice network, the memory load for the linear branch is less than the memory load for the choice branch. If we assume that speakers compute the complexity of the two branches independently rather than computing the complexity of the two possible network descriptions in their entirety, then they are predicted to show the same preferences in Experiment 2 that they showed in Experiment 1. Thus, if the speakers' decision is based only on the relative difficulty of the two branch descriptions rather than the relative difficulty of the two complete network descriptions, then we predict that they will prefer to describe the short branch before the long branch and the linear branch before the choice branch, even when they must end their network descriptions with Circle 1.

\section{EXPERIMENT 2}

The purpose of Experiment 2 was to examine whether the minimal-load principle formulated by Levelt (1981, 1982,1989 ) should be defined over the entire network or over just each branch. 


\section{Method}

Experiment 2 was similar to Experiment 1, but we made one modification to the procedure: The participants were told that they had to begin and end their descriptions with Circle 1. Practice trials were sufficient to make the task clear to all participants. Twentyfour undergraduates from the same participant pool as in Experiment 1 participated in this experiment. Design and data analysis were the same as in Experiment 1.

\section{Results}

Six of the 24 participants consistently chose the same side of every network first (4 participants always went left first, and 2 always went right first). The pattern of data was the same when all 24 participants were included and when only the 18 participants showing a preference were included, and so the reported data include only the 18 participants who varied in their initial choice.

The results are shown in Table 2 . The data clearly replicate the results from Experiment 1. When the two branches were of the same type (i.e., linear-linear or choice-choice), then the participants' decisions were based on number of circles (shorter side described first). When the branches were of different types (linear-choice or choice-linear), then number of circles ceased to matter, and the decision was to describe the linear branch first. Again, the preference for a linear over a choice branch overrides the preference for a shorter over a longer branch.

The statistical results were as follows. Only one main effect was significant: the effect of whether the right side was linear or choice $[F(1,17)=6.22]$. The participants preferred to go right when the right side was linear (.52) and left when it was choice (.35). The effect of whether the left side was linear or choice was in the expected direction (.39 or .48 , respectively), but it was not reliable $[F(1,17)=1.98]$. The effect of number of circles was also in the same direction as in Experiment 1 (.30 when the left side was shorter and .41 when the right side was shorter), but the effect was not reliable $[F(1,17)=2.34]$.

More importantly, the three-way interaction among these three factors was reliable $[F(1,17)=4.97]$. The pattern, which is shown in Table 2, demonstrates that the participants preferred to describe the shorter side first only when the type of branch on each side was the same; otherwise, they preferred to describe the linear branch before the choice one. In other words, the pattern is precisely the one found in Experiment 1. One difference that should perhaps be noted is that the data appear to be

Table 2

The Participants' Decisions to Describe the Left Branch (Values <.50) or Right Branch (Values $>$.50) First in Experiment 2

\begin{tabular}{ccc}
\hline & \multicolumn{2}{c}{ Number of Circles } \\
\cline { 2 - 3 } Branch Type & 3 left, 5 right & 5 left, 3 right \\
\hline Linear-Linear & .34 & .59 \\
Choice-Choice & .24 & .54 \\
Linear-Choice & .35 & .28 \\
Choice-Linear & .59 & .56 \\
\hline
\end{tabular}

Note-SEM $=0.094$ more variable in Experiment 2, as shown by the increased standard error of the mean (Table 2).

We also examined the participants' strategies for returning from the end of the first described branch to the beginning of the second. In contrast to what was found in Experiment 1, in which 22 of 24 participants chose the jumping strategy, a large number of the participants in Experiment 2 chose to step: 10 participants stepped on every single trial, and only 2 were exclusively jumpers. The remaining 12 participants used a combination of jumping, stepping, and a third strategy that included both stepping and jumping on a single trial: The participants using this "hybrid" strategy jumped from the end of Branch 1 to the start of the second, described each circle of Branch 2, and then stepped from the end of Branch 2 back to the network's starting point. Of the 12 participants who used more than one strategy in the experiment, 6 jumped on most trials, 2 stepped on most trials, and 4 used the hybrid strategy most often. Most of these 12 participants seemed to move from strategy to strategy in no predictable fashion, with a couple of exceptions: One participant, who was predominantly a jumper, stepped only on the first three trials and then jumped for the remainder. This pattern was seen in Experiment 1 with 1 of the 2 participants who was not an exclusive jumper. The other participant stepped on the first 28 trials and then used the hybrid strategy on the 29th trial. When this move met with no disapproval from the experimenter, this participant expressed annoyance at the experimenter for not having told him that jumping was acceptable. The participant then jumped on most of the remaining trials. Choice of jumping, stepping, or the hybrid approach did not appear to depend on the type of branch: Each strategy was about as common with linear as with choice branches and with short as with long branches.

Thus, one interesting difference that did emerge between the two experiments is that when the task required returning to the initial return address twice, some participants became more cautious in their description strategy; few risked jumping on every single trial. Another generalization that emerged is that jumping appeared to be the most efficient strategy, as indicated both by the participants' tendency to stick to it once they discovered it and by the annoyance expressed by the participant who discovered jumping only late in the experiment. It also appears, then, that the jumping strategy was less obvious to the participants in Experiment 2 than it was in Experiment 1 . Finally, recall that in Experiment 1, the participant who consistently stepped also appeared to treat each circle in a network as part of a list, in contrast to the other participants who used fuller and more varied utterances. This tendency was not as evident in Experiment 2: The 2 participants who jumped exclusively described the networks in a list-like manner; of the 10 exclusive steppers, only 2 preferred to use clauses, and the rest chose the abbreviated description style. Thus, it is difficult to draw many conclusions concerning what caused the participants to jump or step. It does appear, though, that the 
greater demands imposed on the participants in Experiment 2 (each network required more description and required more maintenance of the initial return address) led the participants to adopt the stepping strategy. Further exploration of this tendency could be fruitful, because it suggests that jumping is an efficient but taxing strategy, whereas stepping is an inefficient but less taxing strategy.

\section{Discussion}

The pattern of data from Experiment 2 concerning which branch the participants chose to describe first is virtually identical to the pattern found in Experiment 1: Linear branches were described before choice branches; if branch type was the same, then short branches were described before long ones. Even though the participants had to begin and end their descriptions of the figures with Circle 2 (the first choice point), they showed the same preferences as they did when they could end their descriptions on the last circle of the second branch.

These results suggest that the second version of the minimal-load principle is correct: Speakers base their decision concerning which branch to describe first on a comparison of the difficulty of the two branches, not a comparison of the difficulty of the two entire network descriptions that result from starting with one branch or the other. Indeed, if the speaker were to perform this latter series of computations, he or she would already have done the work of planning the description-in fact, the speaker would have done the work of planning two descriptions. We propose that, instead, speakers begin the task of describing the networks by computing the complexity of the description of each branch and then choosing the branch associated with the simpler description. Their utterance then begins with the simpler branch. It could be that, in the long run, starting with the simpler branch does not save memory load overall, as in Experiment 2; but this consequence is not available to speakers, because they do not plan that far ahead.

This version of the minimal-load principle is more in the spirit of what Kempen and colleagues (Kempen, 1987; Kempen \& Hoenkamp, 1987) and Levelt and colleagues (Bock \& Levelt, 1994; Levelt, 1989) have called the principle of incrementalism. According to Levelt (1989), in a language production model that is incremental, "all components can work in parallel, but they all work on different bits and pieces of the utterance under construction" (p. 24). For example, speakers need not wait until they have a complete syntactic structure for a sentence before beginning the task of phonological encoding (Bock \& Levelt, 1994). Our suggestion extends incrementalism to the realm of discourse planning, suggesting that speakers do not have to have a complete discourse representation for a network before they begin giving their description.

\section{GENERAL DISCUSSION}

We conducted two experiments, and, for both, the primary dependent variable was whether a speaker chose to describe first the left- or right-hand branch of a twobranch network. In Experiment 1, we found that the participants preferred to describe a short branch before a long branch, and preferred to describe a linear branch before a choice branch. Both of these findings conform to the predictions of the minimal-load principle as originally formulated by Levelt $(1981,1982,1989)$. In addition, the preference for linear branches was stronger than the preference for short branches, as indicated by the participants' tendency to describe a linear branch rather than a choice branch even when the former was longer. This result suggested that the minimal-load principle should be defined over single branches of the networks, not over the entire networks.

In Experiment 2, we required the participants to return to the starting circle during their network descriptions. This manipulation was introduced to test directly whether speakers plan entire network descriptions before beginning to speak or whether they compare only the relative difficulty of the two branches. The results were consistent with the second alternative. This finding implies that discourse planning is incremental, in line with more recent theoretical and experimental work in language production. Thus, the production system does not have to wait for the entire discourse to be planned before beginning articulation (or any of the stages between discourse planning and articulation); instead, the system can organize the material corresponding to the description of the second branch of the network during the articulation of the first.

The results of this study, together with other experimental work, indicate that the language production system has some tendency to order constituents so that easier material is dealt with before more difficult material. This tendency is evident both at the level of word ordering within clauses and at the more macro level of clause ordering within discourses. As briefly mentioned in the introduction, work on word order within clauses has shown that words that are more concrete, frequent, salient, or prototypical, or that are semantically primed tend to occur early in sentences. Similarly, words corresponding to the agent or topic of a sentence tend to occur early. These tendencies can all be taken to reflect a general preference for the most available and accessible word concept to occur early in a sentence. Similarly, the present experiments, as well as Levelt's earlier work, suggest that speakers order clauses so that easier material is described first. It appears that the language production system (perhaps like the human organism itself) likes to postpone more difficult tasks.

Why might the production system operate in this way? We would argue that, for both the speaker and the listener, there are advantages of ordering clauses so that more accessible words in sentences and less complex clauses in discourse are dealt with first. This strategy allows a speaker to begin with the easier material, which requires less planning time. The remainder of the utterance or discourse can then be planned during articulation. In contrast, if the speaker begins with the difficult material, planning must occur during some period of dysfluency 
(during a silent pause, a filled pause, or some other form of hesitation). Thus, a speaker who begins with easier material can make optimal use of scarce processing resources and, at the same time, can avoid producing dysfluent speech (the latter consideration is important if one wishes to hold the floor during a conversation).

Indeed, the bias in the language production system to begin with easier material makes the system more incremental. Consider the following analogy: Imagine that a person has to clean his or her entire house, with rooms varying in their extent of disarray. Our hypothetical housekeeper could proceed in (at least) two ways: One would be to consider the entire task and not begin cleaning until the plan for the entire house had been developed; the other would be to start with a room that requires little planning or work itself and, while cleaning that room, plan the best way to clean the others. The latter strategy has the advantage that it reduces the latency to begin the housekeeping chores, and it allows planning and execution to take place in parallel. Similarly, as Bock has argued (Bock, 1986, 1987a, 1987b; Bock, Loebell, \& Morey, 1992), if the sentence production system places the most available concept into an early sentence position, speaking can begin while the rest of the sentence is being planned. If the discourse production system begins descriptions with easier material, the work of planning the more difficult material can be carried out in parallel with articulation.

It may also be easier for the comprehender if the speaker orders a description so that easier material precedes more difficult material. Consider the networks we used in this study. The listener who has to reconstruct these networks from a verbal description alone must engage in many of the same bookkeeping tasks required for the speaker - for instance, the listener must branch out from choice points and then return to them. It is likely easier for the listener to understand the description if the memory load imposed by these bookkeeping tasks is kept to a minimum. In addition, according to Gernsbacher (1990), it is easier for comprehenders to build up a "mental structure" for some linguistic sequence if it is based on a solid foundation rather than a weaker foundation. The foundation will generally be built from the earlier material in the sequence (see Gernsbacher, 1990, for details). It seems likely that, if the material presented earlier is relatively easy to comprehend, then the foundation for the mental structure will be built more securely, and so the entire representation will be more accurately constructed and ultimately will be more useful to the comprehender.

\section{REFERENCES}

Bock, J. K. (1986). Meaning, sound, and syntax: Lexical priming in sentence production. Journal of Experimental Psychology: Learning, Memory, \& Cognition, 12, 575-586.

BocK, J. K. (1987a). Coordinating words and syntax in speech plans. In A. Ellis (Ed.), Progress in the psychology of language (pp. 337-390). Hillsdale, NJ: Erlbaum.

Bock, J. K. (1987b). An effect of the accessibility of word forms on sentence structures. Journal of Memory \& Language, 26, 119-137.

Bock, J. K., \& LeVeLT, W. J. M. (1994). Language production: Grammatical encoding. In M. A. Gernsbacher (Ed.), Handbook of psycholinguistics (pp. 945-984). New York: Academic Press.

BoCK, J. K., LOEBELL, H., \& MOREY, R. (1992). From conceptual roles to structural relations: Bridging the syntactic cleft. Psychological Review, 99, 150-171.

BOCK, J. K., \& WARREN, R. K. (1985). Conceptual accessibility and syntactic structure in sentence formulation. Cognition, 21, 47-67.

FERREIRA, F. (1994). Choice of passive voice is affected by verb type and animacy. Journal of Memory \& Language, 33, 715-736.

GERNSBACHER, M. A. (1990). Language comprehension as structure building. Hillsdale, $\mathrm{NJ}$ : Erlbaum.

Kelly, M. H., BoCK, J. K., \& KeIL, F. C. (1986). Prototypicality in a linguistic context: Effects on sentence structure. Journal of Memory \& Language, 25, 59-74.

KEMPEN, G. (1987). A framework for incremental syntactic tree formation. In J. McDermott (Ed.), Proceedings of the Tenth International Joint Conference on Artificial Intelligence (pp. 655-660). Los Altos, CA: Morgan Kaufmann.

Kempen, G., \& Hoenkamp, E. (1987). An incremental procedural grammar for sentence formulation. Cognitive Science, 11, 201-258

LEVELT, W. J. M. (1981). The speaker's linearization problem. Philosophical Transactions of the Royal Society of London: Series B, 295, 305315.

LEVELT, W. J. M. (1982). Linearization in describing spatial networks. In S. Peters \& E. Saarinen (Eds.), Processes, beliefs, and questions (pp. 199-220). Dordrecht: D. Reidel.

LEVELT, W. J. M. (1989). Speaking: From intertion to articulation. Cambridge, MA: MIT Press.

LLOYD, P. (1991). Strategies used to communicate route directions by telephone: A comparison of the performance of 7-year-olds, 10-yearolds, and adults. Journal of Child Language, 18, 171-189.

Tombin, R. S. (1983). On the interaction of syntactic subject, thematic information, and agent in English. Journal of Pragmatics, 7, 411-432.

(Manuscript received September 1, 1996; accepted for publication November 12, 1996.) 